\title{
Yangxin Tongmai Formula ameliorates impaired glucose tolerance in children with Graves' disease through upregulation of the insulin receptor levels
}

\author{
Yan-hong $\mathrm{LUO}^{1}$, Min ZHU ${ }^{1}$, Dong-gang WANG ${ }^{1}$, Yu-sheng YANG ${ }^{1}$, Tao TAN², Hua ZHU ${ }^{2}$, Jian-feng $H E^{1, *}$ \\ ${ }^{1}$ Children's Hospital Chongqing Medical University, Chongqing 400000, China; ${ }^{2}$ Department of Surgery, Davis Heart and Lung \\ Research Institute, the Ohio State University Wexner Medical Center, Columbus, OH 43210, USA
}

\begin{abstract}
Graves' disease (GD) is the leading cause of hyperthyroidism, and the majority of GD patients eventually develop disorders of glucose handling, which further affects their quality of life. Yangxin Tongmai formula (YTF) is modified from a famous formula of traditional Chinese medicine for the treatment of cardiovascular diseases. In this study we investigated the potential effects of YTF in the treatment of pediatric GD patients with impaired glucose tolerance. Forty pediatric GD patients and 20 healthy children were recruited for this clinical study. Based on the glucose tolerance, the GD patients were divided into two groups: 20 patients displayed impaired glucose tolerance, while the other 20 patients displayed normal glucose tolerance. YTF was orally administered for 60 days. YTF administration significantly ameliorated the abnormal glucose tolerance and insulin sensitivity in the GD patients with impaired glucose tolerance. To determine the molecular mechanisms of this observation, the number of plasma insulin receptors was determined by ELISA. Before treatment, the fasting and postprandial levels of the insulin receptor were significantly lower in patients with impaired glucose tolerance compared with those in patients with normal glucose tolerance and healthy children. After YTF treatment, both the fasting and the postprandial circulating insulin receptor levels were upregulated, and close to those in healthy children. Therefore, YTF is a potential effective treatment to enhance glucose handling in GD children with impaired glucose tolerance.
\end{abstract}

Keywords: Yangxin Tongmai formula; traditional Chinese medicine; Graves' disease; impaired glucose tolerance; insulin receptor; insulin resistance; Fangjiomics

Acta Pharmacologica Sinica (2018) 39: 923-929; doi: 10.1038/aps.2017.94; published online 23 Nov 2017

\section{Introduction}

Graves' disease (GD) is a common endocrine disorder in pediatric patients and is the leading cause of hyperthyroidism ${ }^{[1,2]}$. The incidence of GD is on the rise, and GD affects both the physical and the mental health of children. Studies by our group and by others have reported that $44 \%-60 \%$ of patients with hyperthyroidism develop abnormal glucose tolerance and glucose metabolism disorders (GMD) ${ }^{[3]}$, which further compromise the quality of life of Graves' disease patients, especially pediatric patients. The current treatments for abnormal glucose tolerance in Graves's patients include exercise, insulin administration and diet control. However, some of the major symptoms of Graves' disease are fatigue, muscle weakness, and heat intolerance ${ }^{[1]}$. In addition, patients usually

\footnotetext{
* To whom correspondence should be addressed.

E-mail 1534568197@qq.com

Received 2017-05-08 Accepted 2017-06-17
}

display insulin insensitivity and insulin antagonism ${ }^{[4-7]}$, which significantly limit the options for treating GMD in Graves' disease patients. Thus, an effective treatment that ameliorates insulin sensitivity and impaired glucose tolerance in Graves' disease patients is highly desired.

It is well-known that the China have a long history of treating diseases that have complex symptoms with Traditional Chinese Medicine (TCM), for which Western medicine treatments usually have limited beneficial effects. Although the ultimate goal of both TCM and Western medicine is to cure diseases, there are fundamental and conceptual differences between TCM and Western medicine. TCM treats the human body and disease as an integrated system, and the crosstalk among different organs and the interplay of various symptoms are all taken into consideration when a TCM doctor diagnoses patients $^{[8-11]}$. In TCM terminology, diabetes is called Xiao-ke, which is caused by an improper diet, emotional disturbances and, most critically, Yin deficiency, in which patients exhibit fatigue, weakness and tiredness. 
There has been two thousand years of history in TCM for the development of an effective treatment for diabetes. As mentioned above, TCM focuses on the human body as an integrated system and treats diseases at their fundamental causes. In this clinical study, we used an established formula, the Yangxin Tongmai Formula (YTF), to treat pediatric Graves' disease patients who had abnormal glucose tolerance. YTF was modified from a famous TCM formula that is used to treat cardiovascular diseases by Professor Zhaokai Yuan at Hunan University of Traditional Chinese Medicine. The original formula of YTF was developed by a renowned TCM physician, Mr Bo-wei QIN, who used it to treat cardiovascular diseases based on the TCM theories of nourishing heart Qi (Tiao Yang Xin Qi) and reestablishing blood circulation (Huo Tong Xue Mai). It uses Renshen (Radix Ginseng) combined with Guizhi (Ramulus Cinnamomi) to invigorate the heart Qi and to warm the blood; Danshen (Radix Salviae Miltiorrhizae) to regulate the meridian and to promote blood circulation to remove blood coagulation; Zhishi (Fructus Aurantii Immaturus) to the break stagnation of qi and to remove food retention, to resolve phlegm and to eliminate mass; and Zexie (Rhizoma Alismatis) to eliminate phlegma-turbidity by promoting dieresis to resolve dampness from the lower energizer. All of these ingredients aim to restore Yin, nourish the heart Qi and reestablish healthy blood circulation, which we believe is potentially suitable for the treatment of diabetes based on the theory of TCM regarding the causes of Xiao-ke (diabetes). In the present study, we used YTF to treat 20 pediatric GD patients who had impaired glucose tolerance for 60 days. Twenty normal children and twenty GD children with normal glucose tolerance were also recruited as controls. Glucose tolerance, insulin sensitivity index (ISI), and plasma insulin levels were measured. We found that plasma glucose, insulin and ISI were significantly increased in GD children with impaired glucose tolerance compared with those in both normal children and GD children with normal glucose tolerance. Interestingly, treatment with YTF significantly decreased the plasma glucose, insulin and ISI levels in GD children with impaired glucose tolerance to the levels of control children. To test the potential mechanism underlying these observations, the plasma insulin receptor levels were quantified by ELISA. We found that the decreased plasma insulin receptor level of GD children with impaired glucose tolerance was restored to the normal range by the treatment with YTF. Thus, our study suggested that YTF could be an effective means to treat GD children with impaired glucose tolerance through the upregulation of the insulin receptor levels and the improvement of insulin sensitivity.

\section{Materials and methods Subjects}

All protocols concerning human subjects were approved by the Reginal Ethical Committee of Children's Hospital Chongqing Medical University, and all investigations with human subjects were conducted with informed consent. GD patients who were recruited into this study were admitted into Children's Hospital Chongqing Medical University from June 2008 to December 2012. The clinical information of patients and normal children is summarized in Table 2. The baseline parameters of all patients and normal individuals are summarized in Supplemental Table S1. The diagnostic criteria for GD was based on that of "Zhufutang Pediatric." All patients had no history of diabetes. Patients who had other symptoms that induced metabolic disorders or diseases were excluded. Forty GD patients were divided into 2 groups: a group of 20 patients who had impaired glucose tolerance (designated as GD1) and a group of 20 patients who had normal glucose tolerance (designated as GD2). There were initially 34 patients recruited into the GD1 group, 11 of whom (2 males and 9 females) were lost during the study, while another 3 female patients did not follow the instruction of taking YTF regularly and were excluded from the study. In addition, 20 healthy children were recruited into the normal group.

\section{Preparation and administration of Yangxin Tongmai Formula (YTF)}

The Yangxin Tongmai Formula (YTF) is composed of 5 crude herbal medicines: $10 \mathrm{~g}$ of Renshen (Radix Ginseng), $15 \mathrm{~g}$ of Danshen (Radix Salviae Miltiorrhizae), $6 \mathrm{~g}$ of Guizhi (Ramulus Cinnamomi), $10 \mathrm{~g}$ of Zhishi (Fructus Aurantii Immaturus), and $10 \mathrm{~g}$ of Zexie (Rhizoma Alismatis). To ensure the quality, accuracy and consistency of the crude herbal components in this study, the individual components were handpicked, checked and weighed by a single board-certified pharmacist and distributed to each patient. The YTF was prepared the night before its administration. Briefly, a mixture of cut crude components (Table 1) was extracted in $50 \mathrm{~mL}$ of distilled water for $30 \mathrm{~min}$ at $>90^{\circ} \mathrm{C}$. The extracted solution was filtered and set aside. The extraction protocol was repeated two further times with the used crude components and combined with the other extraction solutions to yield a total of $150 \mathrm{~mL}$ of the extraction solution. Warm YTF (about $50^{\circ} \mathrm{C}$ ) was orally administered three times per day at a dose of $50 \mathrm{~mL}$ each time. YTF was administered for 60 days. There was an age cutoff criterion: if patients were younger than 7 years old, the dose of YTF was reduced to $50 \%$ of the full dose, and if patients were older than 7 years old, the dose of YTF was the full dose (the same as described above).

Table 1. Components of Yangxin Tongmai Formula (YTF).

\begin{tabular}{llc}
\hline Chinese Pinyin & Latin botanical name & Component ratio (\%) \\
\hline Renshen & Radix Ginseng & $18(10 \mathrm{~g})$ \\
Danshen & Radix Salviae Miltiorrhizae & $27(15 \mathrm{~g})$ \\
Guizhi & Ramulus Cinnamomi & $18(10 \mathrm{~g})$ \\
Zhishi & Fructus Aurantii Immaturus & $18(10 \mathrm{~g})$ \\
Zexie & Rhizoma Alismatis & $18(10 \mathrm{~g})$ \\
\hline
\end{tabular}


Oral glucose tolerance test (OGTT) on pediatric patients and normal children

A glucose tolerance test was performed for the GD patients and the 20 healthy children. Briefly, the children were fasted overnight (from 18:00 to 09:00). Then, a 25\% glucose solution (containing $25 \mathrm{~g}$ of glucose per $100 \mathrm{~mL}$ solution) was freshly prepared by dissolving the glucose in distilled water, and the patients were instructed to drink the glucose solution (at a dose of $1.75 \mathrm{~g} / \mathrm{kg}$ ) within $5 \mathrm{~min}$. Blood samples were taken from the antecubital vein. The blood glucose level was measured by a OneTouch Ultra glucometer (Lifescan, Milpitas, CA, USA). The blood sample was immediately transferred to pyrogen-free blood collection tubes (Bection Dickinson, USA) with EDTA-2Na $(1 \mathrm{mg} / \mathrm{mL})$ and aprotinin $(500 \mathrm{U} / \mathrm{mL})$ and then immediately centrifuged at $1500 \times \mathrm{g}$ for $15 \mathrm{~min}$ at $4^{\circ} \mathrm{C}$. Plasma samples were aliquoted and stored at $-80^{\circ} \mathrm{C}$ until use.

\section{Plasma insulin level measurement}

The plasma insulin levels were measured by a commercial radioimmunoassay (RIA) kit (Shanghai Institute of Biological Products). Assays were carried out according to the manufacturer's instructions with standards run in duplicate and human plasma samples run in triplicate.

\section{Plasma insulin receptor measurement}

The plasma insulin receptor levels were measured by a commercial ELISA kit (Shanghai Yansheng Industrial Co Ltd, Shanghai, China). Assays were carried out according to the manufacturer's instructions with standards run in duplicate and human plasma samples run in triplicate.

Calculation of the insulin sensitivity index (ISI) and homeostasis model assessment index for insulin resistance (HOMA-IR)

The ISI was calculated based on the following formula: ISI= $-1 n /(F P G * F I N S)$. The homeostasis model assessment index for insulin resistance (HOMA-IR) was calculated as fasting insulin (microunits per milliliter) times fasting glucose (millimoles per liter) divided by 22.5. The formula for this is: HOMA-
$\mathrm{IR}=\left(\mathrm{FPG}^{*} \mathrm{FINS}\right) / 22.5$, where FPG and FINS are abbreviations for fast glucose and fast insulin levels, respectively.

\section{Statistical analysis}

Randomized controlled trial and self-control study were used for statistical analysis. The normality and homoscedasticity of the data were tested. The data are shown as the mean \pm SD, and one-way ANOVA was used for multi-group analyses. The SNK test was used to compare two groups if they had an equal variance, while the Tamhane's T2 (M) test was used to compare two groups if equal variance was not assumed. A two-sided test was employed for hypothesis testing. Significance was assumed to be $a=0.05$. The SPSS 17.0 software was used for all statistical analyses.

\section{Results}

Treatment with YTF improves the glucose tolerance of GD1 patients

Between June 2008 and December 2012, 60 children were recruited from the Children's Hospital Chongqing Medical University. Twenty pediatric GD patients with glucose intolerance were designated as the GD1 group, 20 pediatric GD patients with normal glucose tolerance were designated as the GD2 group, and 20 normal children were designated as the control group. The basal clinical characteristics of the three groups of children were similar (except for the GD diagnostic markers) and are summarized in Table 2.

At the beginning of this study, we performed initial oral glucose tolerance tests on all of the children. As shown in Table 3, children in both the control group and the GD2 group showed normal blood glucose levels at basal, $1 \mathrm{~h}$ and $2 \mathrm{~h}$ after the glucose challenge, while patients from the GD1 group had a significantly higher blood glucose level at all three time points compared with those of the control and the GD2 groups. These results suggested that the patients in the GD1 group had impaired glucose tolerance.

To test the potential effects of YTF, patients in the GD1 group were orally administered YTF $(150 \mathrm{~mL} / \mathrm{d}$, for $60 \mathrm{~d})$.

Table 2. Basal clinical characteristics of the three groups in the study. ${ }^{*} P<0.05,{ }^{* *} P<0.01$ compared to control. ${ }^{\# \#} P<0.01$ compared to GD2.

\begin{tabular}{lccl}
\hline \multicolumn{1}{c}{ Parameters (mean \pm SD) } & Control $(n=20)$ & GD1 $(n=20)$ & GD2 $(n=20)$ \\
\hline Male & 5 & 6 & 5 \\
Female & 15 & 14 & 15 \\
Age (year) & $9.77 \pm 3.18$ & $10.45 \pm 2.46$ & $10.61 \pm 2.46$ \\
Body Weight (kg) & $32.88 \pm 13.12$ & $29.38 \pm 8.70$ & $32.60 \pm 11.30$ \\
T3 & $2.31 \pm 0.70$ & $4.02 \pm 1.49^{* *}$ & $4.32 \pm 2.89^{* *}$ \\
T4 & $135.90 \pm 32.95$ & $210.75 \pm 88.20^{* *}$ & $217.50 \pm 83.82^{* * *}$ \\
TSH & $3.98 \pm 1.44$ & $1.81 \pm 2.66^{* *}$ & $2.75 \pm 2.80^{* *}$ \\
Basal blood glucose (mmol/L) & $5.02 \pm 0.44$ & $6.61 \pm 0.28^{* *, \# \#}$ & $5.32 \pm 0.32$ \\
Postprandial blood glucose 60 min (mmol/L) & $6.56 \pm 0.59$ & $8.71 \pm 0.94^{* *, \# \#}$ & $6.71 \pm 0.46$ \\
Postprandial blood glucose 120 min (mmol/L) & $6.64 \pm 0.49$ & $8.91 \pm 0.73^{* *, \# \#}$ & $6.89 \pm 0.45$ \\
\hline
\end{tabular}

GD1 group had impaired glucose tolerance. GD2 group had normal glucose tolerance. 
Table 3. Blood glucose levels. ${ }^{*} P<0.05,{ }^{* *} P<0.01$ compared to control. ${ }^{\# \#} P<0.01$ compared to GD2. ${ }^{\& \&} P<0.01$ compared to GD1 prior to treatment.

\begin{tabular}{lcccc}
\hline \multicolumn{1}{c}{ Group } & Control $(n=20)$ & GD1 group $(n=20)$ & GD2 group $(n=20)$ & GD1(post-treatment) $(n=20)$ \\
\hline Fast & $5.02 \pm 0.44$ & $6.61 \pm 0.28^{* *, \# \#}$ & $5.32 \pm 0.32^{*}$ & $5.40 \pm 0.39^{\text {\&\& }}$ \\
Postprandial $60 \mathrm{~min}$ & $6.56 \pm 0.59$ & $8.71 \pm 0.94^{* *, \# \#}$ & $6.71 \pm 0.46$ & $6.88 \pm 0.49^{\text {\&\& }}$ \\
Postprandial 120 min & $6.64 \pm 0.49$ & $8.91 \pm 0.73^{* *, \#}$ & $6.89 \pm 0.45$ & $7.11 \pm 0.42^{\text {\&\& }}$ \\
\hline
\end{tabular}

GD1 group had impaired glucose tolerance. GD2 group had normal glucose tolerance.

After treatment, OGTT was performed again. As shown in Table 3, treatment with YTF significantly improved glucose tolerance in the GD1 patients. Eighteen out of 20 patients showed improved glucose tolerance after treatment with YTF (Figure 1).

\section{YTF treatment decreases elevated plasma insulin levels in GD1 patients}

It has been well-documented that the impaired glucose tolerance in GD patients is due to insulin insensitivity ${ }^{[4-7]}$. One of the major symptoms that insulin insensitive patients experience is an elevated plasma insulin level. Thus, we first measured both the fasting and the postprandial plasma insulin levels in all of the children. As shown in Table 4, when we compared the fasting plasma insulin levels between the groups, we found that the GD1 group had the highest level, followed by the GD2 group, and that the control group had the lowest level. While there was no significant difference in the fasting insulin levels between the GD1 and the GD2 groups, both the GD1 $(P<0.01)$ and the GD2 $(P<0.05)$ groups had significantly higher fasting plasma insulin levels than the control group. More interestingly, when we analyzed the postprandial plasma insulin levels, we found the same trend

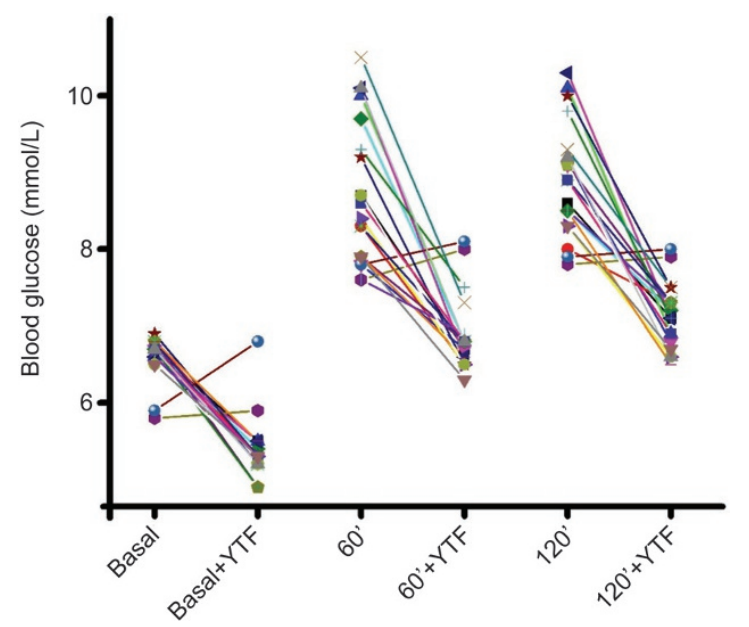

Figure 1. Treatment of YTF reduces both fast and postprandial blood glucose in GD1 patients. Individual data points showed that 18 of 20 GD1 patients responded to treatment of YTF, indicating YTF treatment effectively reduced blood glucose in GD1 patients. Basal: fast blood glucose; 60': postprandial blood glucose at $60 \mathrm{~min}$; 120': postprandial blood glucose at $120 \mathrm{~min}$. as that of the fasting plasma insulin levels. Furthermore, the postprandial plasma insulin level in the GD1 group was significantly higher than that in both the GD2 $(P<0.01)$ and the control $(P<0.01)$ groups. Finally, we found that treatment with YTF could significantly reduce both the fasting (vs GD1 group prior to treatment; $P<0.05$ ) and postprandial (vs GD1 group prior to treatment; $P<0.01)$ plasma insulin levels in patients of the GD2 group, although they were still higher than those of the control group $(P<0.05)$.

\section{Treatment with YTF improves ISI and HOMA-IR in GD1 patients}

In addition, ISI and homeostasis model assessment index for insulin resistance (HOMA-IR) are two key clinical parameters that demonstrate the insulin sensitivity of the patients. Similar to the findings for the plasma insulin levels, we found that both the ISI and the HOMA-IR of GD1 patients were significantly worse than those of the children in the GD2 $(P<0.05)$ and the control $(P<0.01)$ groups. After YTF treatment, we observed a significant improvement in both the ISI (vs GD1 prior to treatment; $P<0.05$ ) and the HOMA-IR (vs GD1 prior to treatment; $P<0.05)$ (Table 5).

Treatment with YTF improves insulin sensitivity through the upregulation of the insulin receptor levels

There are many factors that contribute to insulin insensitivity. The reduction of the components of the insulin pathway is a critical factor, especially the reduction of insulin receptors $^{[12]}$. To explore the molecular mechanisms underlying the insulin insensitivity that is observed in GD1 patients with impaired glucose tolerance, we measured the insulin receptor levels by ELISA. As shown in Table 6, although there were no significant differences in the fasting insulin receptor levels among the three groups, there was a trend that showed that the control group had the highest insulin receptor level, followed by the GD2 group, with the GD1 group having the lowest insulin receptor level. Moreover, the postprandial insulin receptor level in the GD1 group was significantly decreased compared with the levels in the control group and the GD2 group $(P<0.01)$. More importantly, YTF treatment significantly increased both the fasting and the postprandial insulin receptor levels in the GD1 group (compared with the GD1 group prior to treatment; $P<0.01$ ) to levels that were similar to those of the control group. Another interesting observation from this study was that the postprandial circulating IR levels remained stable in the control and the GD2 groups; however, the postprandial IR level in the GD1 group signifi- 
Table 4. Plasma insulin levels. ${ }^{* *} P<0.01$ compared to control. ${ }^{\# \# P<0.05}$ as compared to GD1 post-treatment. ${ }^{\& \&} P<0.01$ as compared to GD2. ${ }^{\$ \$} P<0.01 F$ test.

\begin{tabular}{lclcc}
\hline \multicolumn{1}{c}{ Group } & Control $(n=20)$ & GD1 group $(n=20)$ & GD2 group $(n=20)$ & GD1 (post-treatment) $(n=20)$ \\
\hline Fast & $18.03 \pm 2.69$ & $22.07 \pm 3.43^{* *}$ & $20.46 \pm 2.58^{* *}$ & $20.31 \pm 2.38^{* *}$ \\
Postprandial 60 min & $44.58 \pm 3.89$ & $73.92 \pm 4.65^{* *, \#, \text { \&\& }}$ & $52.17 \pm 4.64^{* *}$ & $7.02^{\$ \$}$ \\
\hline
\end{tabular}

GD1 group had impaired glucose tolerance. GD2 group had normal glucose tolerance.

Table 5. ISI and HOMA-IR values. ${ }^{*} P<0.05$ compared to control. ${ }^{\#} P<0.05$ compared to GD1 post-treatment. ${ }^{\& \&} P<0.01$ compared to GD2. ${ }^{\$ \$} P<0.01 F$ test.

\begin{tabular}{lcccc}
\hline Group & Normal $(n=20)$ & GD1 group $(n=20)$ & GD2 group $(n=20)$ & GD1 (post-treatment) $(n=20)$ \\
\hline ISI & $-4.55 \pm 0.17$ & $-4.99 \pm 0.19^{*, \#, \text { \& \& }}$ & $-4.69 \pm 0.15^{*}$ & $-4.69 \pm 0.11^{*}$ \\
HOMA-IR & $4.26 \pm 0.74$ & $6.48 \pm 1.05^{*, \#, \text { \& }}$ & $4.86 \pm 0.69^{*}$ & $27.57^{\$ \$}$ \\
\hline
\end{tabular}

GD1 group had impaired glucose tolerance. GD2 group had normal glucose tolerance.

Table 6. Insulin receptor (IR) levels. ${ }^{* *} P<0.01$ as compared to control. ${ }^{\# \#} P<0.01$ compared to fast IR in same group. ${ }^{\text {\&\&}} P<0.01$ compared to GD1 posttreatment. ${ }^{\$} P<0.01$ as compared to GD2.

\begin{tabular}{lllcc}
\hline \multicolumn{1}{c}{ Group } & Control $(n=20)$ & GD1 group $(n=20)$ & GD2 group $(n=20)$ & GD1 (post-treatment) $(n=20)$ \\
\hline Fast IR & $115.17 \pm 65.97$ & $80.56 \pm 37.10$ & $97.31 \pm 60.46$ & $110.21 \pm 53.26$ \\
Postprandial IR & $114.14 \pm 61.60$ & $56.82 \pm 23.84^{* *, \# \text {, \& }}$ & $97.56 \pm 43.16$ & $105.76 \pm 45.94$ \\
\hline
\end{tabular}

GD1 group had impaired glucose tolerance. GD2 group had normal glucose tolerance.

cantly decreased $(P<0.01)$ compared with the fasting IR level, indicating that the decrease of IR might contribute to glucose intolerance in the GD1 group. Interestingly, treatment with YTF not only upregulated the fasting IR level but also upregulated the postprandial IR level in the GD1 group. Therefore, our data suggest that YTF ameliorated the insulin sensitivity of the human body through the upregulation of insulin receptor levels.

In summary, our clinical study revealed that treatment with YTF could improve multiple critical parameters in patients of the GD1 group to enhance their glucose handling, including effectively lowering their blood glucose and insulin levels and upregulating their IR levels (especially their postprandial IR levels), and could enhance ISI and HOMA-IR.

\section{Discussion}

YTF and the underlying Fangjiomics theory is an ideal choice and an effective means for the treatment of GD patients with impaired glucose tolerance

The present study originated from a common dilemma in Western medicine practice, in which physicians have known that more than $50 \%$ of GD patients have or will develop impaired blood glucose, however, there is no effective treatment for this patient population. The reasons for this are: first, due to the symptoms of GD, patients are incapable of exercising to control their blood glucose; second, GD patients are usually insulin-resistant, so it is extremely difficult and even dangerous to use single-target treatment, such as insulin, to lower their blood glucose (hypoglycemia induced by insulin is a huge concern in GD patients).

Given this background, TCM treats the human body as an integrated system and usually has a superior effect in the treatment of (or can even cure) the disease. In the present study, we first observed that our GD patient cohort displayed impaired glucose tolerance, and the likely cause of this symptom was reduced insulin sensitivity, as evidenced by the impaired ISI and HOMA-IR. Based on the theory of TCM, this symptom is due to Yin deficiency and entangled Qi. Therefore, YTF, which enhances the balance of Yin and Yang and which promotes blood circulation, will improve this symptom in patients. Indeed, when we treated patients with TYF for 60 days, one of our most exciting findings was that both the fasting and the postprandial plasma insulin levels in the GD1 group of patients were significantly reduced together with the reduction of blood glucose levels. This is completely different from treatment with Western medicine. In the case of the YTF treatment, we treated the disease from its "root", which, translated into Western medicine terms, is to effectively enhance the whole body's insulin sensitivity. Our ELISA assay confirmed that the insulin receptor, a key molecular for regulating insulin sensitivity in the body, was significantly upregulated by the YTF treatment, which means that the YTF treatment makes the patients responsive to insulin from their own body again (no need for an insulin injection). We 
Table 7. YTF treatment significantly improve glucose handling in GD1 patients.

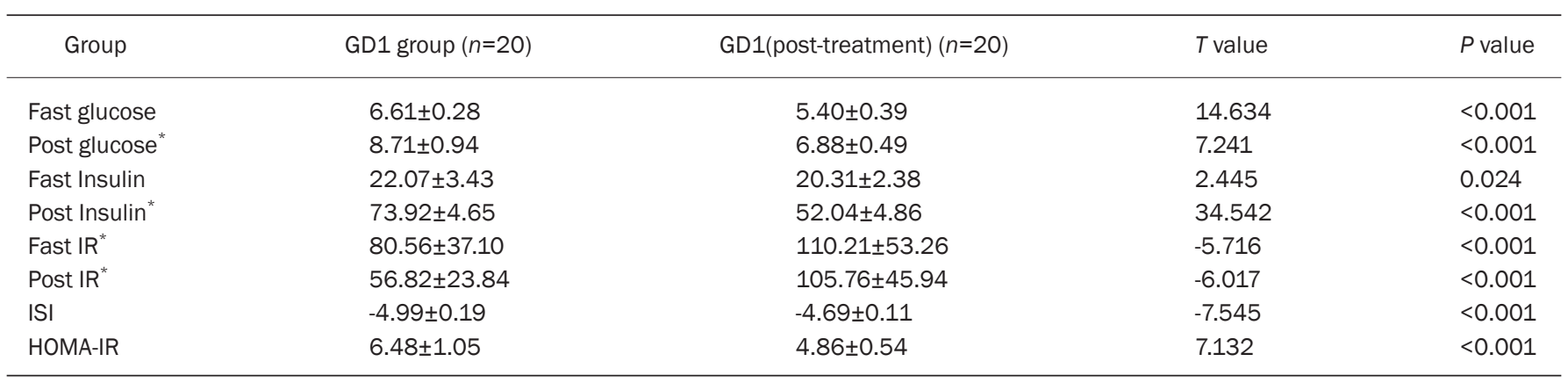

Post glucose: postprandial glucose; Post Insulin: postprandial insulin level; Fast IR: fast insulin receptor level; Post IR: postprandial insulin receptor level.

believe that this is the ultimate goal of Fangjiomics ${ }^{[8-11]}$, which takes advantage of TCM to promote systematic reactions in the human body to actually cure the disease and not only treat the symptoms. In fact, other groups have followed the guidance of Fangjiomics to explore the molecular actions of the Traditional Chinese Herbal Formula for the treatment of diabetes $^{[13]}$. I believe that this is a promising direction of TCM research.

\section{Molecular mechanisms of YTF in the treatment of GD patients with impaired glucose tolerance}

Many previous studies have proposed different causes for impaired glucose tolerance in GD patients. Some studies have proposed that the enhancement of gastrointestinal motility causes an upregulation of blood glucose levels due to increased glucose absorption via the digestive tract, which is called oxyhyperglycemia-type postprandial hyperglycemia ${ }^{[14,15]}$. Other studies have attributed the glucose intolerance in GD patients to increased endogenous gluconeogenesis (due to the excess of thyroid hormone $)^{[16,17]}$ and insulin resistance ${ }^{[18,19]}$. Studies have indicated that insulin receptors are associated with insulin metabolism and influence the psychological effects of insulin, so decreasing the amount of insulin receptors and lowering their affinity may induce insulin resistance. Furthermore, the decrease in postprandial insulin receptors is one of the most important steps in the pathogenesis of glucose metabolism disorder and insulin resistance. Consistent with previous studies, we found that both the fasting and the postprandial IR levels were significantly reduced in the patients of the GD1 group compared with those in the patients of both the normal control group and the GD2 group. More significantly, the patients from the GD1 group had even less IR available postprandially, which is when IR is normally required. This observation might explain the insulin insensitivity that was seen in the patients of the GD1 group and serves as a marker to evaluate the effects of potential treatments. Indeed, we found that, following treatment with YTF, the IR levels of the patients in the GD1 group were restored to the levels of normal individuals (higher than the patients in the GD2 group). This result might be suggestive of one of the molecular actions of YTF for improving glucose handling in patients.
YTF was formed by the experience of the famous modern Traditional Chinese Medicine practitioner Mr Bo-wei QIN for the treatment of cardiovascular disease by nourishing the heart $Q i$ and promoting blood circulation. Yuan et al have studied hyperlipidemia in insulin resistance syndrome (IRS) patients and showed that treatment with Yangxin Tongmai tablets could lead to a reduction in blood lipid and fasting insulin levels and a significant decrease in body mass index, while it could lead to an increase in the insulin sensitivity index (ISI). Huang et al studied this mechanism and showed that after treatment, the number of high affinity erythrocyte insulin receptors (EIR-H) and low affinity erythrocyte insulin receptors (EIR-L) of an insulin resistance syndrome (IRS) patient significantly increased after the treatment in both the Yangxin Tongmai tablets group and the metformin tablets group. The function of Yangxin Tongmai tablets and metformin tablets in treating insulin resistance syndrome (IRS) may be related to the following factors: enhancing ATPase activity, decreasing $\mathrm{Ca}^{2+}$, inhibiting $\mathrm{Ca}^{2+}$ overload and resisting lipid peroxidation of the erythrocyte membrane ${ }^{[20]}$. Huang et al studied the effect of Yangxin Tongmai tablets on insulin receptors in insulin resistance syndrome (IRS) in a rat model and identified that, when IRS is present, the density of insulin receptors and their affinity showed significant decreases and that the effect of the Yangxin Tongmai tablets on the increase of EIR is better than that of the metformin tablets ${ }^{[21]}$. Yangxin Tongmai tablets have a good effect in treating insulin resistance syndrome (IRS) ${ }^{[22]}$. GD patients with impaired glucose tolerance were treated with YTF for 2 months, and their 60-min fasting and postprandial blood glucose levels and fasting and postprandial insulin levels decreased, while their fasting and postprandial insulin receptor levels and ISI increased. Furthermore, their HOMA-IR decreased. The above-mentioned indexes had no significant differences between the GD1 (post-treatment) group and the GD2 group. Indicators of fasting and postprandial insulin levels, ISI and HOMA-IR in the GD1 post-treatment group showed significant differences compared with the normal group.

Overall, our current study and previous studies have once again emphasized the multiple target effects of YTF in the treatment of GD patients with impaired glucose tolerance. It 
may upregulate insulin receptors, avoid the low density of insulin receptors, which is induced by the feeble binding affinity of insulin, and promote the bioactivity of insulin.

\section{Future directions}

One study cannot address all aspects of the biological actions of YTF in the treatment of GD patients with impaired glucose tolerance. Several questions should be addressed in future studies: first, will YTF be effective for the treatment of GD patients in general? Based on the TCM theory, hyperthyroidism is caused by a Yin deficiency of multiple internal organs, including the heart, kidney, liver and spleen. Thus, the recommended treatments include nourishing the heart and kidney, removing heat from the heart and liver, and then restoring the $Q i$ and balance between $Y i n$ and Yang. Therefore, it suggests that YTF, which aims to nourish the heart and to restore $Q i$, might also be effective for the treatment of GD. We are currently recruiting patients in this direction. Second, are there more targets of YTF besides the insulin receptor? As mentioned above, multiple factors have been linked to the development of glucose intolerance in GD patients. While our study has suggested that the effect of YTF was to enhance insulin sensitivity by upregulating insulin receptor levels, there might be further actions of YTF. For example, can YTF reduce glucose absorption from the intestine or can it reduce glycogen biogenesis? All of these possibilities require more studies and are the exact purpose of Fangjiomics.

In sum, the current clinical study indicated that TCM might be an ideal choice for the treatment of chronic endocrine disease. The Fangjiomics approach is a promising direction for identifying the potential targets of the TCM treatments.

\section{Author contribution}

Yan-hong LUO participated in the manuscript writing and experimental design and performed the experiments; Min ZHU, Dong-gang WANG, and Yu-sheng YANG participated in performing parts of the experiments; Tao TAN participated in the manuscript writing, manuscript editing and data analysis; Hua ZHU and Jian-feng HE participated in the manuscript writing, experimental design and data analysis. All authors have read and approved the manuscript.

\section{References}

1 Smith TJ, Hegedus L. Graves' Disease. N Engl J Med 2016; 375: 1552-65.

2 Smith DM, Dutta S, Ahmed F, Thaha MA. Change in practice over four decades in the management of Graves' disease in Scotland. J Thyroid Res 2016; 2016: 9697849.

3 Doar JW, Stamp TC, Wynn V, Audhya TK. Effects of oral and intravenous glucose loading in thyrotoxicosis. Studies of plasma glucose, free fatty acid, plasma insulin and blood pyruvate levels. Diabetes 1969; 18: 633-9.

4 Kabadi UM, Eisenstein AB. Impaired pancreatic alpha-cell response in hyperthyroidism. J Clin Endocrinol Metab 1980; 51: 478-82.

5 Cohen P, Barzilai N, Barzilai D, Karnieli E. Correlation between insulin clearance and insulin responsiveness: studies in normal, obese, hyperthyroid, and Cushing's syndrome patients. Metabolism 1986; 35: 744-9.

6 Shen DC, Davidson MB, Kuo SW, Sheu WH. Peripheral and hepatic insulin antagonism in hyperthyroidism. J Clin Endocrinol Metab 1988; 66: 565-9.

7 Ohguni S, Notsu K, Kato Y. Correlation of plasma free thyroxine levels with insulin sensitivity and metabolic clearance rate of insulin in patients with hyperthyroid Graves' disease. Intern Med 1995; 34 : 339-41.

8 Wang Z, Duan DD, Wang YY. Editorial: combination therapy of vascular diseases and fangjiomics: when west meets east in the era of phenomics. Curr Vasc Pharmacol 2015; 13: 420-2.

9 Duan DD, Wang Z, Zhang BL, Wang YY. Fangjiomics: revealing adaptive omics pharmacological mechanisms of the myriad combination therapies to achieve personalized medicine. Acta Pharmacol Sin 2015; 36: 651-3.

10 Liu J, Wang Z. Diverse array-designed modes of combination therapies in Fangjiomics. Acta Pharmacol Sin 2015; 36: 680-8.

11 Wang Z, Liu J, Cheng Y, Wang Y. Fangjiomics: in search of effective and safe combination therapies. J Clin Pharmacol 2011; 51: 113251.

12 Friedman JE, Ishizuka T, Liu S, Farrell CJ, Bedol D, Koletsky RJ, et al. Reduced insulin receptor signaling in the obese spontaneously hypertensive Koletsky rat. Am J Physiol 1997; 273: E1014-23.

13 Yang ZZ, Liu W, Zhang F, Li Z, Cheng YY. Deciphering the therapeutic mechanisms of Xiao-Ke-An in treatment of type 2 diabetes in mice by a Fangjiomics approach. Acta Pharmacol Sin 2015; 36: 699-707.

14 Ahren B. Hyperthyroidism and glucose intolerance. Acta Med Scandinav 1986; 22: 5-14.

15 Levin RJ, Smyth DH. The effect of the thyroid gland on intestinal absorption of hexoses. J Physiol 1963; 169: 755-69.

16 Hellstrom L, Wahrenberg H, Reynisdottir S, Arner P. Catecholamineinduced adipocyte lipolysis in human hyperthyroidism. J Clin Endocrinol Metab 1997; 82: 159-66.

17 Vaughan $M$. An in vitro effect of triiodothyronine on rat adipose tissue. J Clin Invest 1967; 46: 1482-91.

18 Dimitriadis G, Mitrou P, Lambadiari V, Boutati E, Maratou E, Koukkou $\mathrm{E}$, et al. Glucose and lipid fluxes in the adipose tissue after meal ingestion in hyperthyroidism. J Clin Endocrinol Metab 2006; 91: 1112-8.

19 Bloomgarden ZT. American Diabetes Association Annual Meeting, 1999: insulin action and the development of type 2 diabetes. Diabetes Care 2000; 23: 248-52.

20 Huang XP, Yuan ZK, Lu FG, Xie MZ, Hu ZX, Yao XL. Effects of Yangxin Tongmai Tablets on erythrocyte membrane of insulin resistance syndrome. Chin J Information Tradit Chin Med 2003; 10: 12-4.

21 Huang XP, Yuan ZK, Lu FG. Effects of Yangxin Tongmai Tablet on rythrocyte insulin receptor of insulin resistance syndrome in rats. Chin J Cardiovasc Rev 2004; 2: 220-3.

22 Hu ZX, Yuan ZK, Huang XP, Lu FG, Xie MZ. An experimental study on Yangxin tongmai tablet to insulin resistance syndrome in rats, hemorheology and insulin sensitivity. Chin J Integr Med Cardio-/ Cerebrovasc Dis 2003; 1: 255-7. 